\title{
Ion Channels in Bone Morphogenetic Protein Signaling
}

\author{
Laura Faith George, BSc, Trevor Isner, BA, and Emily Anne Bates, PhD
}

\begin{abstract}
How a single fertilized egg develops into a complex multicellular organism is one of the great mysteries of life. Developmental biology textbooks describe cascades of ligands, receptors, kinases, and transcription factors that designate proliferation, migration, and ultimately fate of cells organized into a multicellular organism. Recently, it has become apparent that ion channels are integral to the process of developmental signaling. Ion channels provide bioelectric signals that must intersect with the known developmental signaling pathways. We review some evidence that bioelectric signaling contributes to bone morphogenetic protein signaling.
\end{abstract}

Keywords: channelopathies, bone morphogenetic protein, BMP, inwardly rectifying potassium channels, ion channels and pumps, bioelectric signaling

\section{Introduction}

$\mathbf{O}$ NE OF LIFE'S GREAT MYSTERIES is how cells communicate to orchestrate movement, division, and cell fate choices to generate a complex multicellular organism. An elegant genetic screen in Drosophila melanogaster revealed signaling cascades that include ligands, receptors, kinases, and transcription factors that are essential for segmentation and patterning of an embryo. ${ }^{1}$ Upon these foundational discoveries, developmental biologists have assembled conserved networks of interactions that are essential for patterning a multicellular organism. Textbooks and thousands of research articles detail the undeniable contributions of components of canonical developmental signaling pathways.

More recently it has become apparent that bioelectric signals work together with these canonical developmental signals for cell-cell communication to correctly specify morphogenesis. Bioelectric signals are created by variations in ion channel and ion pump activity and establish the cellular transmembrane potential (Vmem). Foundational studies from Michael Levin's laboratory have shown that the bioelectric pattern of cells within a developing tissue is important for the pattern and size of the developing tissue. ${ }^{2-5}$ Changing this bioelectric pattern can result in drastic changes in morphological development as profound as the formation of ectopic eyes in Xenopus laevis. ${ }^{6}$ Changes in the transmembrane potential pattern also affect mammalian craniofacial development, suggesting that bioelectric signals play a conserved role in morphological development. ${ }^{7}$

\section{Ion Channels in Human Development}

Morphological features associated with mutations found in human patients suggest that the role for ion channels in de- velopment is conserved. Mutations that disrupt $\mathrm{KCNJ} 2$, encoding the inwardly rectifying potassium channel Kir2.1, cause morphological abnormalities such as cleft palate, micrognathia (small jaw), hypertelorism, low set ears, and digital defects such as syndactyly, clinodactyly, and brachydactyly. ${ }^{8,9}$ Mutations that disrupt the Cav1.2 (CACNA1C) calcium channel are associated with similar craniofacial and digital abnormalities in Timothy's syndrome. ${ }^{10}$ Mutations that disrupt another inwardly rectifying potassium channel called GIRK2 (KCNJ6) are associated with abnormal craniofacial development, including high arched palate, microcephaly, and lipodysorophy. ${ }^{11}$ Mutations that disrupt a voltage-gated potassium channel Kv10.1 (Eag, $\mathrm{KCNH} 1)$ are associated with stereotypical facial features, clinodactyly, and hypoplasia or aplasia of fingernails and toenails. $^{12,13}$ Mutations that disrupt a two-pore potassium channel called TASK3 (KCNK9) are associated with a higharched or cleft palate, short mandible, high eyebrows, a narrow forehead, a short philtrum, and cleft soft palate as part of Birk-Barel syndrome. ${ }^{14,15}$ Gain of function and loss of function mutations in the TRPV4 channel are associated with skeletal dysplasias. ${ }^{16-19}$ Although not all of these mutations that disrupt ion channel function have been shown to be causative, the fact that disruption of homologous ion channels in more than one organism is associated with morphological abnormalities suggests that these mutations contribute to the morphological defects.

\section{Ion Channels in Drosophila Development}

A screen in D. melanogaster has revealed that a wide variety of ion channels are involved in developmental patterning. ${ }^{20}$ Of 177 ion channels screened in Drosophila, 44

Department of Pediatrics, University of Colorado School of Medicine, Aurora, Colorado. 
significantly contributed to wing development. ${ }^{20}$ The high number of channels identified to contribute to morphogenesis in this screen suggests that ion channels likely play a broad role in patterning. Interestingly, ion channels of many different types including calcium, sodium, potassium, chloride, and ligand-gated ion channels were among those identified in the screen, revealing that the ion channels that impinge upon developmental pathways are not limited to a single category. ${ }^{20}$

Many of the wing phenotypes observed upon disruption of the 44 ion channels identified in this screen were mild, possibly because ion channels often belong to families that are able to compensate for each other when a single member is disrupted. When one channel is deleted or knocked down, other channels can become upregulated in response, masking the phenotypes of the channel disruption. ${ }^{21,22}$ Indeed, within the 44 identified, we found that ion channels belonging to smaller families had more severe phenotypes than those belonging to larger families that could potentially be compensating for each other. ${ }^{20}$ The ability of ion channels to compensate for each other means that the number of ion channels involved in development may be even higher than this screen revealed, as it is possible that compensation may mask the effects of single channel disruptions.

\section{The Ion Channel Kir2.1 and Bone Morphogenetic Protein Signaling}

Understanding how the bioelectric signals intersect with established signaling pathways is a key question. Some clues can be taken from a mouse lacking the inwardly rectifying potassium channel, Kir2.1, encoded by the gene Kcnj2. Several lines of evidence suggest that Kir2.1 function may specifically impact bone morphogenetic protein (BMP) signaling. The BMPs signaling pathway is among the essential conserved developmental cues that are used repeatedly throughout morphological development. Work in Drosophila lends insight into the mechanism by which Irk channels may impact BMP signaling. BMPs such as Drosophila Dpp are members of the transforming growth factor (TGF)- $\beta$ superfamily and follow a stereotypical signaling cascade. ${ }^{23} \mathrm{BMP}$ ligands are released from the BMP-producing cells. BMP ligands bind to a complex of type 1 and type 2 serine threonine kinase receptors. ${ }^{24}$ Upon ligand binding, the type 2 receptor activates the type 1 receptor. ${ }^{25}$ The activated type 1 receptor phosphorylates receptor-associated Smads (1/5/8), which interact with Smad 4 and translocate into the nucleus to affect target gene expression. ${ }^{26-28}$

Results consistent with a link between Kir2.1 and BMP family members are compelling. First, the Kir2.1 knockout mouse $K c n j 2^{K O / K O}$ has phenotypes that are consistent with the channel's involvement in BMP signaling. For example, similar to mice in which BMP signaling components have been removed from the cranial neural crest, Kcnj2 $2^{K O / K O}$ mice have hypoplastic maxilla, hypoplastic mandibles lacking the coronoid process, a cleft palate, and hypoplastic frontal bones leaving an enlarged fontanelle. ${ }^{29-31}$ Loss of Kir2.1 leads to similar limb patterning defects as loss of BMP ligands from the limb buds. ${ }^{32,33}$

Second, Kir2.1 and BMP signaling are both required in the cranial neural crest cells for the proliferation of the mesenchyme of the palatal shelves. ${ }^{29,34,35}$ Third, E13.5 Kcnj2 $2^{K O / K O}$ craniofacial tissues show a reduction in read-outs of BMP signaling such as phosphorylation of Smads1/5/8 and expression of BMP transcriptional targets. ${ }^{29}$ However, TGF- $\beta$ signaling seems to be intact in Kcnj2 $2^{K O / K O}$ tissue. ${ }^{29}$ Furthermore, inhibition of Kir2.1 orthologs (Irk channels) in Drosophila also leads to phenotypes reminiscent of loss of a Drosophila BMP called Dpp..$^{21,25,36-38}$ Inhibition or loss of Irk channels also reduces Drosophila Smad phosphorylation and expression of a Dpp transcriptional target. ${ }^{21}$ Together these lines of evidence suggest that Kir/Irk channels are important for efficient BMP signaling.

\section{Mechanism of Kir2.1 Effect on BMP Signaling}

Irk channels are required in the Dpp-producing cells of the Drosophila wing disk, but not required in the Dppresponding cells for efficient Dpp signaling. ${ }^{33}$ Inhibition of Irk channels in the Dpp-producing cells does not reduce the amount of Dpp produced. ${ }^{33}$ However, without the channel's function, the Dpp is not released in a regulated pulsatile manner, suggesting that Irk channels regulate the release of Dpp. ${ }^{33}$ In neurons, Irk channels regulate the transmembrane potential, which, in turn, affects intracellular calcium levels that can drive neurotransmitter release. ${ }^{39}$ Inhibition of Irk channels reduces the amplitude and duration of native increases in intracellular calcium in Dpp-producing cells in the Drosophila wing disk, suggesting that Dpp release may be regulated by ion channel function and intracellular calcium levels. ${ }^{33}$ BMP acts noncell autonomously. The ligand cannot perform its function if it is not released properly. If similar mechanisms regulate BMP and neurotransmitter release, a role for multiple types of ion channels in morphogenesis becomes apparent. It remains to be determined whether this model applies to other ion channels.

It is clear that ion channels contribute to morphological development of structures that we do not consider to be composed of excitable cells. The mechanism by which bioelectric signals intersect with canonical developmental signaling pathways is an area with exciting open questions.

\section{Author Disclosure Statement}

No competing financial interests exist.

\section{References}

1. Nusslein-Volhard C, Wieschaus E. Mutations affecting segment number and polarity in Drosophila. Nature 1980;287: 795-801.

2. Levin M. Molecular bioelectricity in developmental biology: New tools and recent discoveries: Control of cell behavior and pattern formation by transmembrane potential gradients. Bioessays 2012;34:205-217.

3. Levin M. Endogenous bioelectrical networks store nongenetic patterning information during development and regeneration. J Physiol 2014;592:2295-2305.

4. Levin M. Molecular bioelectricity: How endogenous voltage potentials control cell behavior and instruct pattern regulation in vivo. Mol Biol Cell 2014;25:3835-3850.

5. Levin M, Pezzulo G, Finkelstein JM. Endogenous bioelectric signaling networks: exploiting voltage gradients for control of growth and form. Annu Rev Biomed Eng 2017; 19:353-387.

6. Pai VP, Aw S, Shomrat T, et al. Transmembrane voltage potential controls embryonic eye patterning in Xenopus laevis. Development 2012;139:313-323. 
7. Adams DS, Uzel SG, Akagi J, et al. Bioelectric signalling via potassium channels: A mechanism for craniofacial dysmorphogenesis in KCNJ2-associated Andersen-Tawil Syndrome. J Physiol 2016;594:3245-3270.

8. Plaster NM, Tawil R, Tristani-Firouzi M, et al. Mutations in Kir2.1 cause the developmental and episodic electrical phenotypes of Andersen's syndrome. Cell 2001;105:511-519.

9. Yoon G, Oberoi S, Tristani-Firouzi M, et al. AndersenTawil syndrome: Prospective cohort analysis and expansion of the phenotype. Am J Med Genet A 2006;140:312-321.

10. Splawski I, Timothy KW, Sharpe LM, et al. Ca(V)1.2 calcium channel dysfunction causes a multisystem disorder including arrhythmia and autism. Cell 2004;119:19-31.

11. Masotti A, Uva P, Davis-Keppen L, et al. Keppen-Lubinsky syndrome is caused by mutations in the inwardly rectifying K+ channel encoded by KCNJ6. Am J Hum Genet 2015;96: 295-300.

12. Simons C, Rash LD, Crawford J, et al. Corrigendum: Mutations in the voltage-gated potassium channel gene KCNH1 cause Temple-Baraitser syndrome and epilepsy. Nat Genet 2015;47:304.

13. Simons C, Rash LD, Crawford J, et al. Mutations in the voltage-gated potassium channel gene KCNH1 cause Temple-Baraitser syndrome and epilepsy. Nat Genet 2015; 47:73-77.

14. Barel O, Shalev SA, Ofir R, et al. Maternally inherited Birk Barel mental retardation dysmorphism syndrome caused by a mutation in the genomically imprinted potassium channel KCNK9. Am J Hum Genet 2008;83:193-199.

15. Graham JM, Jr., Zadeh N, Kelley M, et al. KCNK9 imprinting syndrome-further delineation of a possible treatable disorder. Am J Med Genet A 2016;170:2632-2637.

16. Krakow D, Vriens J, Camacho N, et al. Mutations in the gene encoding the calcium-permeable ion channel TRPV4 produce spondylometaphyseal dysplasia, Kozlowski type and metatropic dysplasia. Am J Hum Genet 2009;84:307-315.

17. Masuyama R, Vriens J, Voets T, et al. TRPV4-mediated calcium influx regulates terminal differentiation of osteoclasts. Cell Metab 2008;8:257-265.

18. Nilius B, Voets T. The puzzle of TRPV4 channelopathies. EMBO Rep 2013;14:152-163.

19. Rock MJ, Prenen J, Funari VA, et al. Gain-of-function mutations in TRPV4 cause autosomal dominant brachyolmia. Nat Genet 2008;40:999-1003.

20. George LF, Pradhan SJ, Mitchell D, et al. Ion channel contributions to wing development in Drosophila melanogaster. G3 (Bethesda) 2019;pii: g3.400028.2019.

21. Dahal GR, Rawson J, Gassaway B, et al. An inwardly rectifying $\mathrm{K}+$ channel is required for patterning. Development 2012;139:3653-3664.

22. Rosati B, McKinnon D. Regulation of ion channel expression. Circ Res 2004;94:874-883.

23. Raftery LA, Sutherland DJ. TGF-beta family signal transduction in Drosophila development: From Mad to Smads. Dev Biol 1999;210:251-268.

24. Ruberte E, Marty T, Nellen D, et al. An absolute requirement for both the type II and type I receptors, punt and thick veins, for dpp signaling in vivo. Cell 1995;80:889-897.

25. Letsou A, Arora K, Wrana JL, et al. Drosophila Dpp signaling is mediated by the punt gene product: A dual ligand- binding type II receptor of the TGF beta receptor family. Cell 1995;80:899-908.

26. Heldin $\mathrm{CH}$, Miyazono $\mathrm{K}$, ten Dijke P. TGF-beta signalling from cell membrane to nucleus through SMAD proteins. Nature 1997;390:465-471.

27. Newfeld SJ, Mehra A, Singer MA, et al. Mothers against dpp participates in a DDP/TGF-beta responsive serinethreonine kinase signal transduction cascade. Development 1997;124:3167-3176.

28. Raftery LA, Twombly V, Wharton K, et al. Genetic screens to identify elements of the decapentaplegic signaling pathway in Drosophila. Genetics 1995;139:241-254.

29. Belus MT, Rogers MA, Elzubeir A, et al. Kir2.1 is important for efficient BMP signaling in mammalian face development. Dev Biol 2018;pii: S0012-1606(17)30829-1.

30. Bonilla-Claudio M, Wang J, Bai Y, et al. Bmp signaling regulates a dose-dependent transcriptional program to control facial skeletal development. Development 2012; 139:709-719.

31. Saito H, Yamamura K, Suzuki N. Reduced bone morphogenetic protein receptor type 1A signaling in neural-crestderived cells causes facial dysmorphism. Dis Model Mech 2012;5:948-955.

32. Bandyopadhyay A, Tsuji K, Cox K, et al. Genetic analysis of the roles of BMP2, BMP4, and BMP7 in limb patterning and skeletogenesis. PLoS Genet 2006;2:e216.

33. Dahal GR, Pradhan SJ, Bates EA. Inwardly rectifying potassium channels regulate Dpp release in the Drosophila wing disc. Development 2017.

34. Li L, Wang Y, Lin M, et al. Augmented BMPRIAmediated BMP signaling in cranial neural crest lineage leads to cleft palate formation and delayed tooth differentiation. PLoS One 2013;8:e66107.

35. Liu W, Sun X, Braut A, et al. Distinct functions for Bmp signaling in lip and palate fusion in mice. Development 2005;132:1453-1461.

36. Adachi-Yamada T, Fujimura-Kamada K, Nishida Y, et al. Distortion of proximodistal information causes JNKdependent apoptosis in Drosophila wing. Nature 1999;400: 166-169.

37. de Celis JF. Expression and function of decapentaplegic and thick veins during the differentiation of the veins in the Drosophila wing. Development 1997;124:1007-1018.

38. Zecca M, Basler K, Struhl G. Sequential organizing activities of engrailed, hedgehog and decapentaplegic in the Drosophila wing. Development 1995;121:2265-2278.

39. Perez MF, White FJ, Hu XT. Dopamine D(2) receptor modulation of $\mathrm{K}(+)$ channel activity regulates excitability of nucleus accumbens neurons at different membrane potentials. J Neurophysiol 2006;96:2217-2228.

Address correspondence to: Emily Anne Bates

Department of Pediatrics

University of Colorado School of Medicine 12800 East 19th Avenue, RC 21 North Mail Stop 8313 Aurora, CO 80045

E-mail: Emily.Bates@UCDenver.edu 